\title{
New CAD System for Designing the Pressure Differential Flowmeters
}

\author{
Prof. Dr. Y. Pistun, Assoc. Prof. Dr. L. Lesovoy and PhD R. Fedoryshyn \\ Lviv Polytechnic National University, 12 S. Bandery St., Lviv, 79013, Ukraine, \\ romanfedoryshyn@yahoo.com
}

\begin{abstract}
:
New CAD system is developed in order to provide computer aided design of pressure differential flowmeters for fluid energy carriers and to simplify implementation of new normative documents (ISO 5167.1,2,3,4-2003 and GOST 8.586.1,2,3,4,5-2005). This program meets the requirements of the new Standards and provides accomplishment of the following tasks: verification of conditions (constraints) for application of the pressure differential method according to the requirements of new Standards; calculation of parameters of primary device, pipe straight lengths and the whole flowmeter according to the requirements of new Standards; calculation of uncertainty of results of fluid flowrate and volume measurement; design of optimal flowmeter as to the accuracy of measurement.
\end{abstract}

Key words: Flowmeter, differential pressure, design, accuracy, optimization.

\section{Introduction}

The world prices for the energy resources have been rising up till now, which was caused by the world market trends and by other factors. The Ukrainian situation is complicated by the fact that major part of the most important energy carriers (natural gas and oil) need to be purchased abroad (from Russia, Kazakhstan, Turkmenistan etc). Such a state of things is putting forth a very important task - the task of energy carrier saving.

Efficient consumption and saving of energy is possible only if metering is carried out with high accuracy and on every step and branch of supply of energy carriers. The accuracy of such a metering is defined by technical base, normative base, metrological base and the professional level of personnel.

\section{Normative Base for Flowrate Measurement}

In order to control consumption of fluid energy carriers (natural gas, hot water, overheated steam etc.) the pressure differential method is applied. This method is used for measurement of energy carrier flowrate and volume in pipes with internal diameter of $50 \mathrm{~mm}$ and more.

Metering of fluid energy carriers by means of pressure differential devices in Europe is carried out according to the requirements of ISO $5167.1,2,3,4$ 2003 [1,2,3,4]. In CIS countries the new Intergovernmental Standard GOST 8.586.1,2,3,4,5$2005[5,6,7,8,9]$ is in force. The first four parts of this Intergovernmental Standard were developed as modified versions of ISO 5167.1,2,3,4-2003 and the fifth part of GOST 8.586.5-2005 covers the requirements of $\mathrm{CIS}$ laws on measurement of energy carrier flowrate and volume and normalizes the procedure for measurements.

The Intergovernmental Standard GOST 8.586.1, $2,3,4,5-2005$ was developed by authors of this paper together with scientists and specialists from "Ukrmetrteststandard" National Standardization Body (Ukraine) and with experts from "Gazmetrologia" Field Metrology Centre of "Gazprom" Joint-Stock Company (Russia). This Standard was implemented in Russia in 2007 as a national Standard. In 2010 this Standard was implemented in Ukraine as DSTU GOST 8.586.1,2,3,4,5-2009.

The most important thing here is that based on the analysis we carried out we can say that implementation of the new Standard will provide improvement of accuracy of energy carrier metering.

It should be mentioned that the new Standards in force (ISO 5167.1,2,3,4-2003 and GOST 8.586.1, $2,3,4,5-2005)$ differ from previous Standards considerably. The most significant distinctions are new limitations for application of the pressure differential method, new mathematical formulae for main coefficients of flowrate equation, new requirements to pipe straight lengths and fittings, new requirements to application of flow straighteners and flow conditioners and finally new methodology for assessment of uncertainty of results of flowrate and volume measurement. The detailed comparative analysis of various normative documents on fluid flowrate measurement by means of the pressure differential method is given in [10].

An important distinction between GOST 8.586.1, $2,3,4,5-2005$ and ISO $5167.1,2,3,4-2003$ is that the first one covers a wider area of application of the pressure differential method. In particular there is a possibility to take into account the roughness of pipe 
internal surface by introducing the corresponding correction coefficient into the flowrate equation. In a similar way there is a possibility to take into account other influencing factors. Additionally there is normalization of fluid volume measurement in pressure differential flowmeters as well as normalization of uncertainties of fluid flowrate and volume measurement in GOST 8.586.1,2,3,4,5-2005.

\section{Peculiarities in Implementation of the New Standards}

Naturally such significant distinctions between the new Standards and the previous ones cause considerable difficulties during implementation of the first. In particular, for every flowmeter at least the following tasks have to be accomplished:

- verification of conditions (constraints) for application of the pressure differential method according to the requirements of the new Standards;

- verification of pipe straight lengths according to the requirements of the new Standards;

- calculation of parameters of primary device, pipe straight lengths and flowmeter in general according to the requirements of the new Standards;

- calculation of uncertainty of results of fluid flowrate and volume measurement according to the requirements of the new Standards.

When accomplishing these tasks it is very important to carry out the design of a flowmeter (and of a whole metering system) optimal as to the measuring accuracy i.e. flowmeter providing minimum uncertainty of fluid flowrate and volume measurement.
The methodology of the mentioned above verifications, calculations and design of pressure differential flowmeters was not formalized even for previous Standards. Such methodology is developed by us according to new Standards.

\section{Description of the New CAD System}

In order to simplify accomplishment of the mentioned above tasks and implementation of new Standards (ISO 5167.1,2,3,4-2003 and GOST 8.586.1,2,3,4,5-2005) we propose to accomplish the developed methodology of calculations and design of flowmeters by means of a specialized computer program: system for computer aided calculation and design of pressure differential flowmeters.

Such a system was developed by us and its name is "Raskhod-RU" CAD [11]. By means of this computer program all the mentioned above tasks of verifications, calculations and design of flowmeters can be accomplished. "Raskhod-RU" CAD meets the requirements of new Standards and this computer program is certified in Ukraine and in Russia.

"Raskhod-RU" CAD is intended for design of flowmeters for 48 fluids including the following: natural gas, humid oil gas, overheated steam, dry saturated steam, water, air, nitrogen, argon, oxygen and other. All valid procedures for calculation of expansibility factor for natural gas (NX19 mod.; GERG-91 mod.; AGA8-92DC mod.; VNIC SMV; SD 7-2005) are implemented in the program.

At present "Raskhod-RU" CAD is available in Ukrainian, Russian, Kazakh and English.

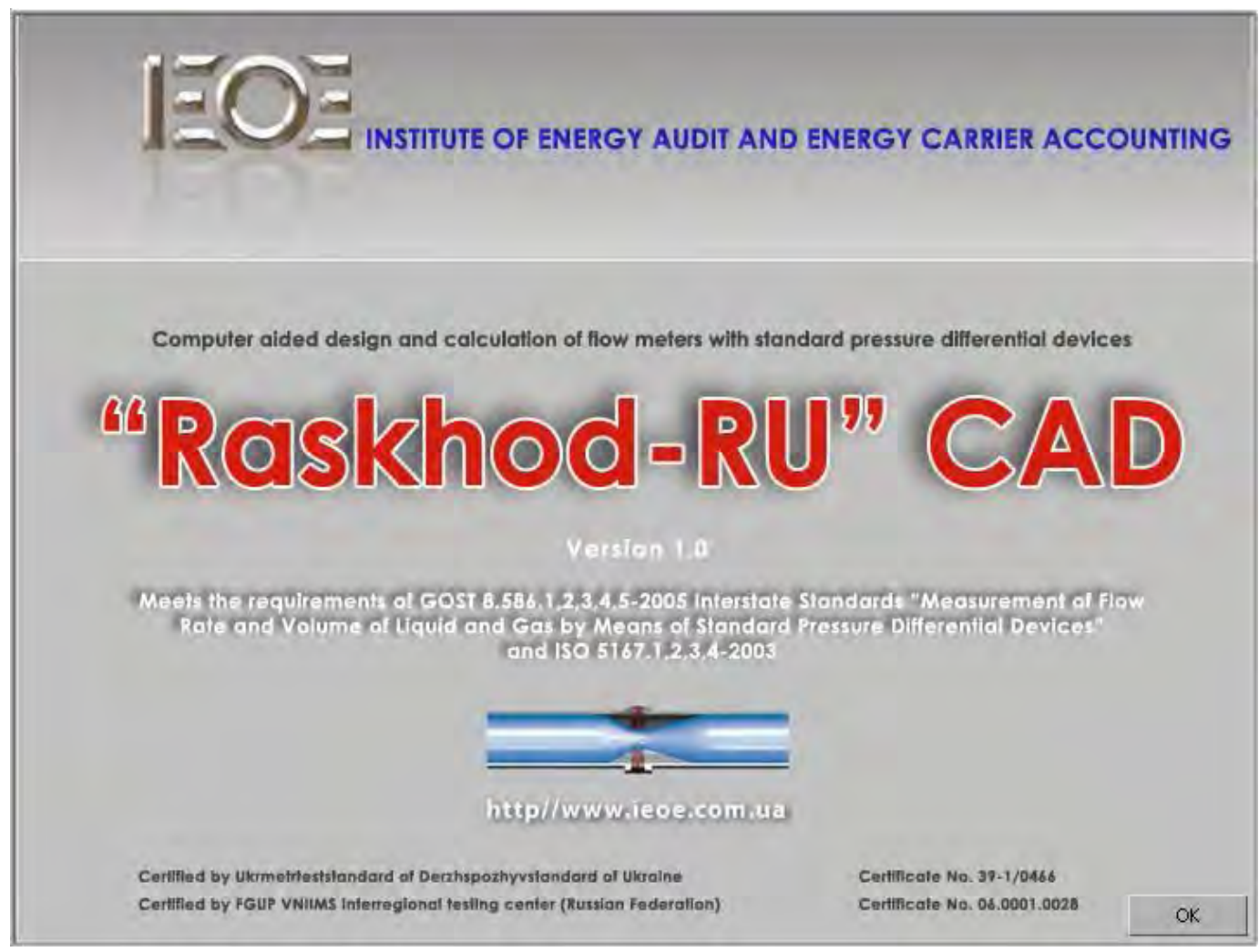

Fig. 1. The first dialog box of "Raskhod-RU" CAD. 
The following four different types of calculation of flowmeter parameters can be accomplished by means of "Raskhod-RU" CAD:

- calculation of flowmeter parameters for the given upper limit of differential pressure measurement;

- calculation of flowmeter parameters for the given allowable pressure loss at primary device;

- calculation of flowmeter parameters to secure minimum uncertainty of flowrate measurement;

- calculation of flowmeter parameters for the given specifications of primary device and pipe (the so called inverse calculation of flowmeter).

The first three types of calculation are applied at initial design of a pressure differential flowmeter. Here in second and third types of calculation an optimization problem is solved to minimize pressure losses at primary device or to minimize the uncertainty of flowrate measurement respectively. And the fourth type of calculation is applied to define the parameters of an existing flowmeter and the conditions of its application according to the requirements of new Standards (ISO 5167.1,2,3,42003 and GOST 8.586.1,2,3,4,5-2005).

When designing a flowmeter the "Raskhod-RU" CAD gives the possibility to choose one of the three possible configurations of the flowmeter:

- with separate measurement of flow parameters;

- with flowrate and volume calculator;

- with measuring complex.

Here the program provides a possibility to specify the parameters of up to four measuring transducers (installed in series) in each channel of measurement of a flow parameter (fluid pressure and temperature and differential pressure across the primary device). In order to provide a wider range of flowrate measurement there is a possibility to specify the parameters of additional measuring transducers (installed in parallel) in the channels of differential pressure measurement and fluid pressure measurement.

After choosing the type of the problem to be solved (type of calculation) the following input data should be entered (see Fig. 2):

- parameters and characteristics of the fluid;

- parameters and characteristics of the primary device;

- parameters and characteristics of the pipe;

- parameters and characteristics of the pipe straight lengths and fittings;

- parameters and characteristics of the measuring and calculating instruments;

- parameters and characteristics of the flowmeter unit.

The part of the program for entering the parameters and characteristics of the fluid consists of the fields where the input data should be entered (the list of the input parameters and characteristics is individual for each type of fluid). The parameters and characteristics for natural gas including the method for calculating the gas compressibility factor (NX 19 mod.) are shown on Fig. 2.

The part of the program for entering the parameters and characteristics of the primary device (orifice plate with corner pressure tappings in this example) is shown on Fig. 3. The list of the input parameters and characteristics is individual for each type of primary device and pressure tappings. The drawing of the primary device appears in the dialog box after choosing the type of the primary device. All the types of standard primary devices according to ISO 5167.1,2,3,4-2003 are included in "Raskhod-RU" CAD. The similar dialog boxes are made for other parameters and characteristics of the flowmeter.

It should be mentioned that calculation and design of a differential pressure flowmeter is a multi-optional problem.

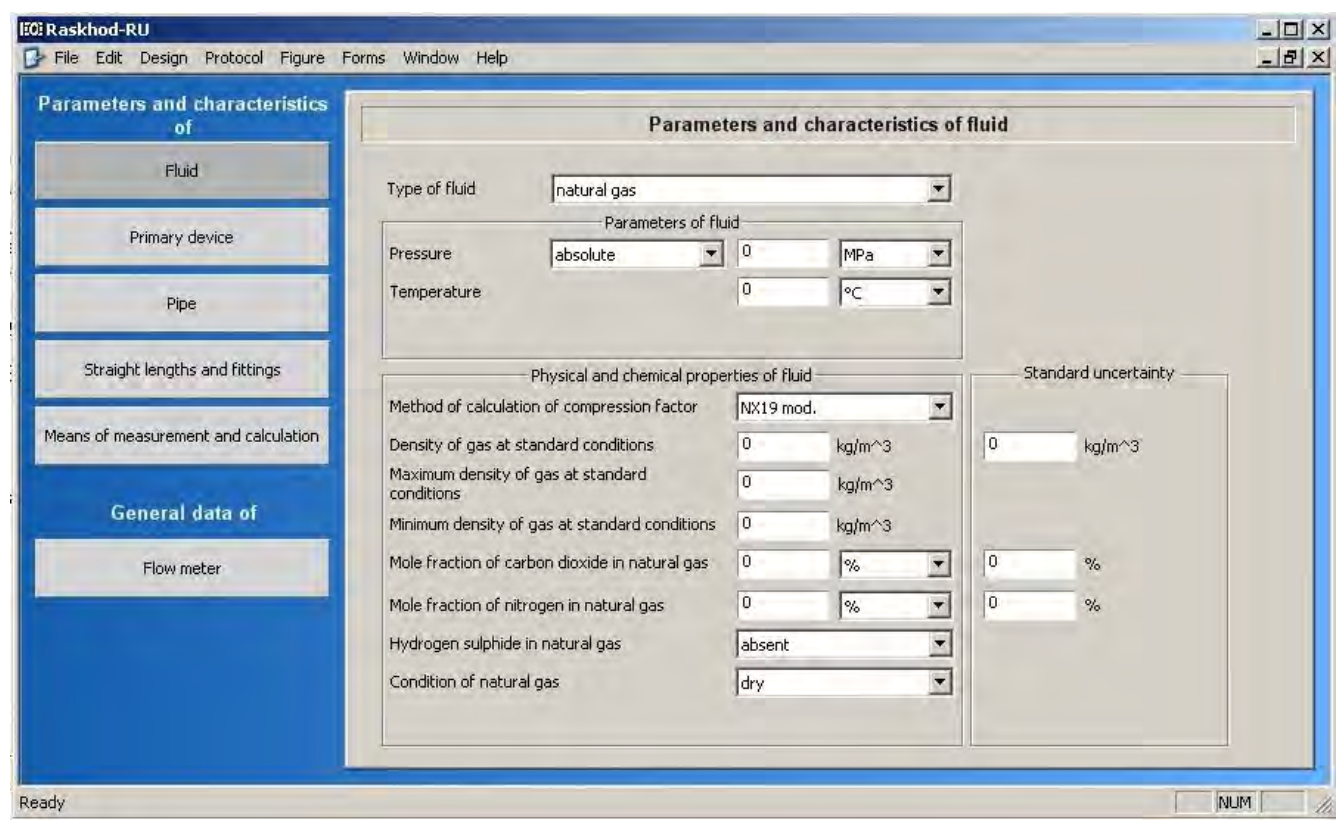

Fig. 2. Dialog box for entering the parameters and characteristics of the fluid (natural gas in this example). 


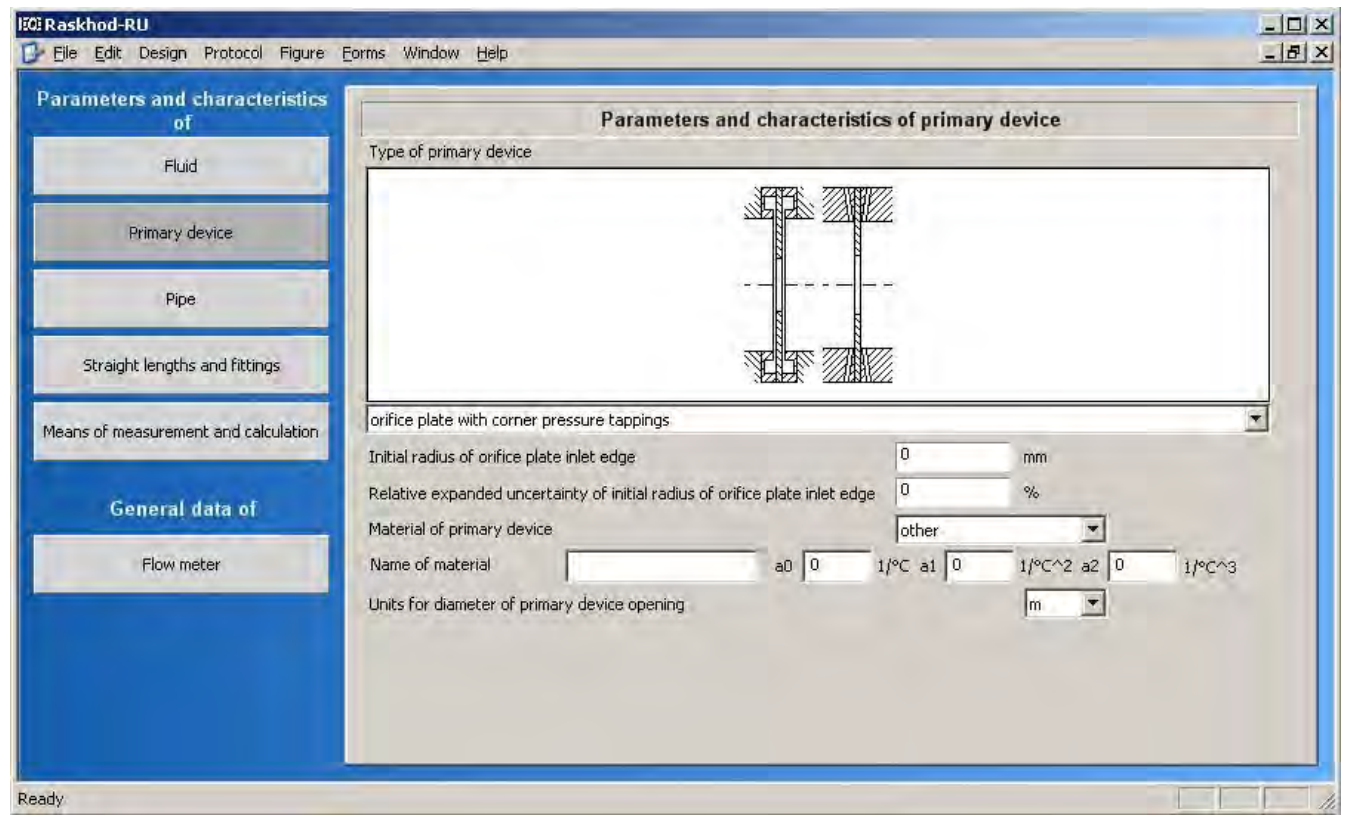

Fig. 3. Dialog box for entering the parameters and characteristics of primary device (orifice plate in this example).

"Raskhod-RU" CAD is an interactive program where each step of the user is being analyzed and in case of a mistake or any wrong input data the warnings, tips or recommendations are returned. The most important tips are those concerning inconformance to the requirements of new Standards. In this case "RaskhodRU" CAD is like an educational software for teaching the requirements of new Standards and for teaching the design of a pressure differential flowmeter.
The interactive recommendations of "Raskhod-RU" CAD provide the possibility not only to design a pressure differential flowmeter according to the requirements of new Standards but to minimize the uncertainty of flowrate measurement. These recommendations should be taken into account by the user and the input data should be corrected according to the recommendations.

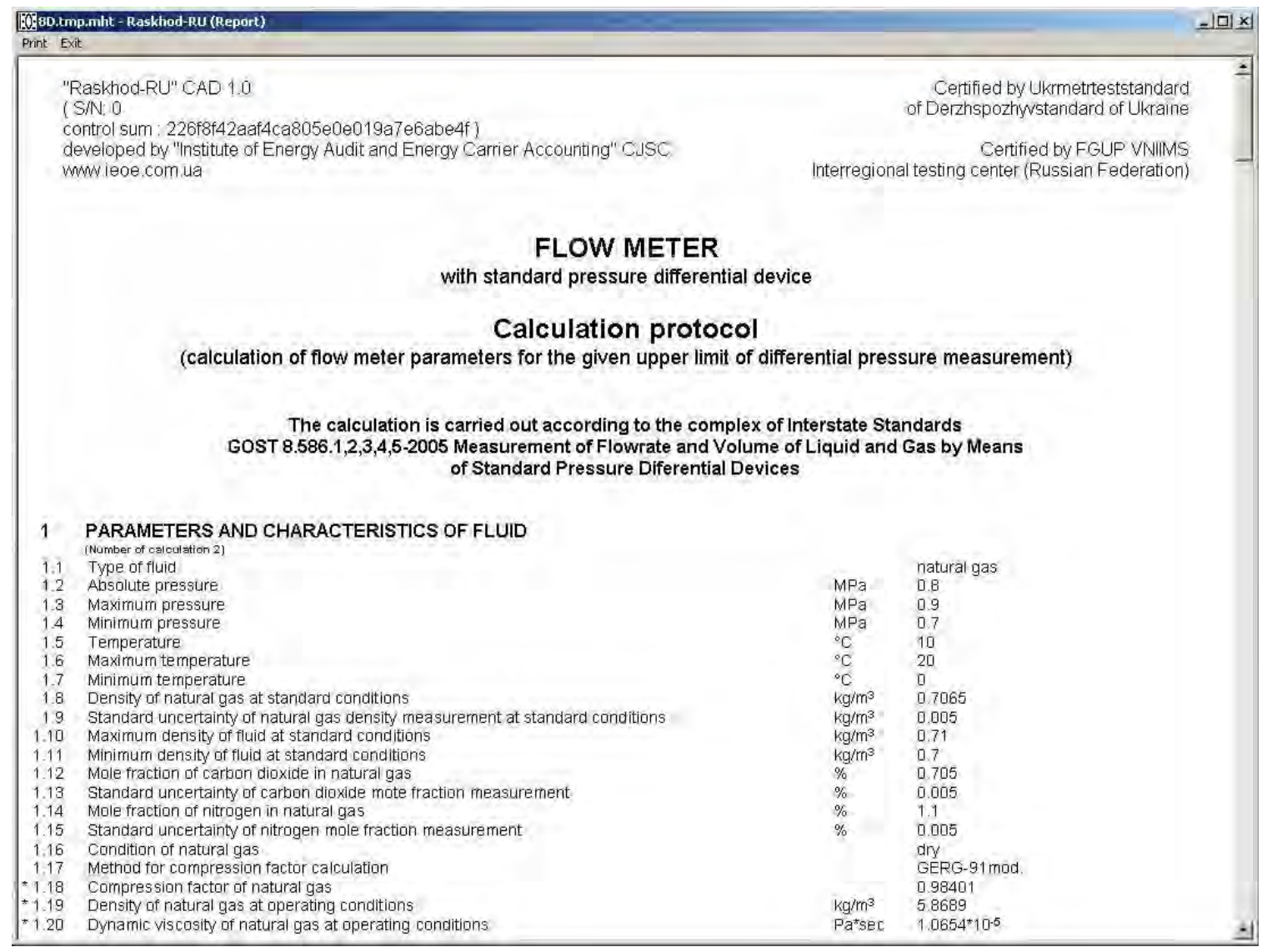

Fig. 4. Flowmeter calculation protocol. 
At the end of such an iterative process the calculation protocol for a differential pressure flowmeter which meets the requirements of the new Standards is returned by the program (see Fig. 4). We can get such a protocol without iterations if all the input data are correct. In any case the "Raskhod-RU" CAD would not return a calculation protocol for a flowmeter which does not meet the requirements of the new Standards.

\section{Advantages of the New CAD System}

The following advantages of "Raskhod-RU" CAD should be specified:

- user friendly interface of the program providing quick and easy learning of possibilities of the program and maximum using of these possibilities which is achieved by logical and understandable distribution of input data among the dialogue boxes; the interface of the program is made in such a way that the dialog boxes are being changed during entering the input data (the fields for the unnecessary parameters and characteristics are taken away if needed);
- interactive shell for data input which is especially important for pipe straight lengths, in particular there is a possibility to use flow straighteners and flow conditioners in the pipe as well as various options for mounting the thermometer;

- automatic control of dialogue boxes during entering the input data by the user which provides avoiding unforeseen mistakes;

- possibility to save the input data files and no possibility to save the calculation protocol (in order to make distortions in the protocol impossible);

- impossibility to carry out calculation and design and to get a calculation protocol for a flowmeter which does not meet the requirements of the new Standards;

- display of warnings about additional component errors of measurement and possible ways to eliminate these errors according to the requirements of new Standards;

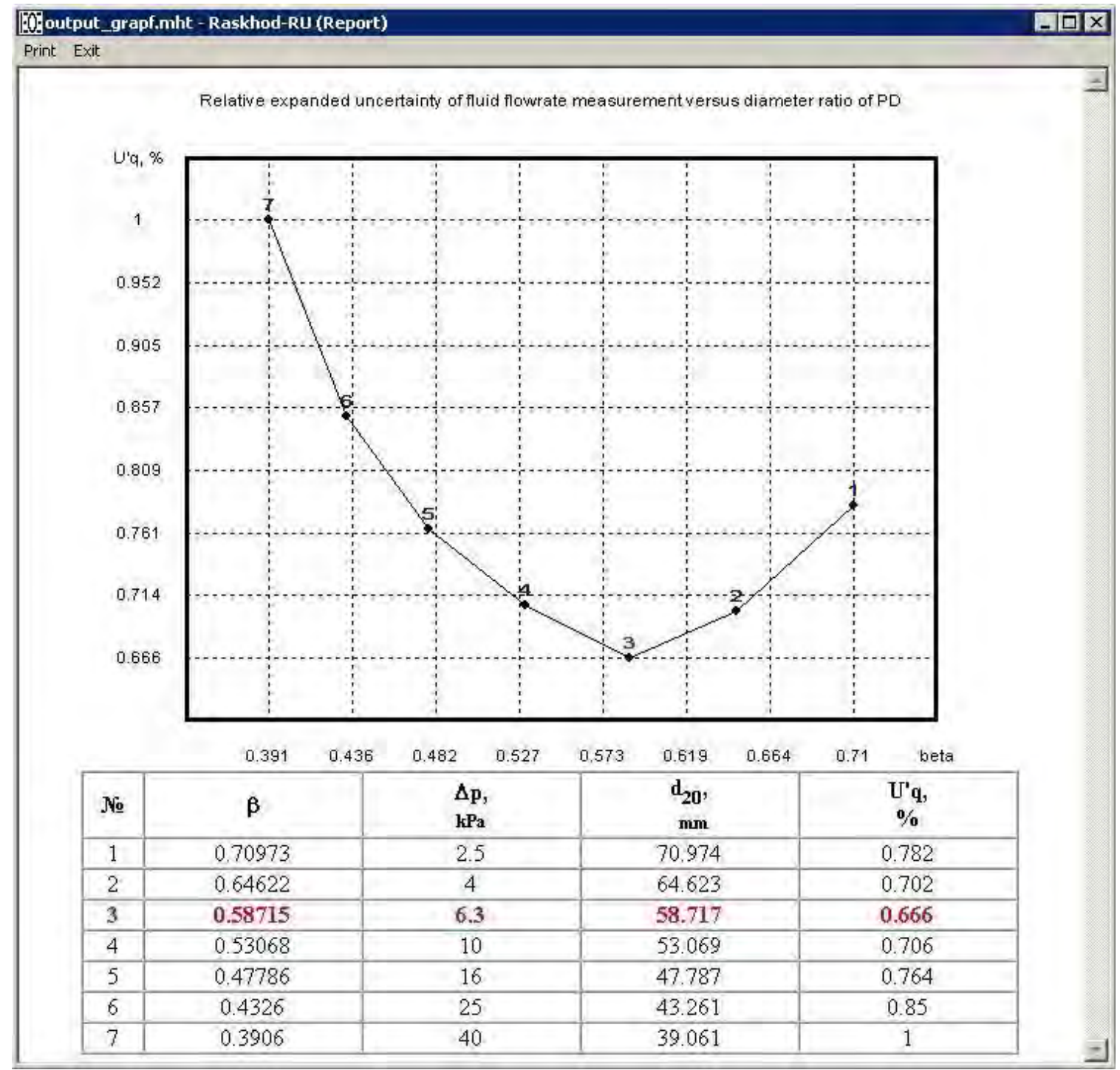

Fig. 5. Curve of relative expanded uncertainty of fluid flowrate measurement versus diameter ratio of primary device. 
- high quality visualization of calculation results which simplifies the analysis and application of the results, in particular there are curves of flowrate measurement uncertainty versus flowrate or other parameters; there are also drawings of primary devices, pipe straight lengths and fittings etc.;

- reliable protection against unauthorized use of the program.

A particularly important advantage of "Raskhod-RU" $C A D$ is the possibility to carry out design of a flowmeter optimal as to the accuracy of flowrate measurement. As an example the curve of relative expanded uncertainty of fluid flowrate measurement versus diameter ratio of primary device is shown on Fig. 5. There is a table under the curve with these values together with upper limits of differential pressure measurement and with diameter of the primary device opening. The presented curve and table demonstrate the possibility of obtaining maximum accuracy of flowrate measurement when designing a pressure differential flowmeter. In the given example the highest accuracy is obtained for flowmeter number three.

No mater what type of calculation is chosen by the user the calculation results are returned in the following way. After all the input data were entered the "Design" should be selected in the top menu of the program (see Fig. 1, Fig. 2). The main calculation results will be displayed in the same dialog box. After selecting "Protocol" in the top menu of the program the calculation protocol with all the input parameters and characteristics together with calculation results and with drawings will be displayed in a separate window (see. Fig. 3). After selecting "Figure" the curve of relative expanded uncertainty of fluid flowrate measurement versus flowrate (or versus diameter ratio of the primary device in case of making the design of a flowmeter optimal as to the accuracy of flowrate measurement) will be displayed in the same dialog box. The calculation results can be printed out.

\section{Conclusions}

The methodology for calculation and design of pressure differential flowmeters is developed by us according to the requirements of the new Standards (ISO 5167.1,2,3,4-2003 and GOST 8.586.1,2,3,4,52005). This methodology is implemented in "Raskhod-RU" CAD which enables computer aided calculation and design of pressure differential flowmeters. At the same time verification of conditions (constraints) for application of the pressure differential method according to the requirements of the new Standards can be carried out by this computer program which simplifies the process of implementation and application of the new Standards significantly. Application of optimal flowmeters as to the accuracy of flowrate measurement, proposed by "Raskhod-RU" CAD, will provide improvement of accuracy of fluid energy carrier metering.

\section{Acknowledgements}

The research has been supported by Institute of Energy Audit and Energy Carrier Accounting.

\section{References}

[1] ISO 5167-1:2003. Measurement of fluid flow by means of pressure differential devices inserted in circular cross-section conduits running full - Part 1: general principles and requirements

[2] ISO 5167-2:2003. Measurement of fluid flow by means of pressure differential devices inserted in circular cross-section conduits running full - Part 2: Orifice plates

[3] ISO 5167-3:2003. Measurement of fluid flow by means of pressure differential devices inserted in circular cross-section conduits running full - Part 3: Nozzles and Venturi nozzles

[4] ISO 5167-4:2003. Measurement of fluid flow by means of pressure differential devices inserted in circular cross-section conduits running full - Part 4: Venturi tubes

[5] GOST 8.586.1-2005 (ISO 5167-1:2003) Measurement of Flowrate and Volume of Liquid and Gas by Means of Standard Pressure Differential Devices - Part 1. Principle of method of measurement and general requirements

[6] GOST 8.586.2-2005 (ISO 5167-2:2003) Measurement of Flowrate and Volume of Liquid and Gas by Means of Standard Pressure Differential Devices - Part 2. Orifice plates. Technical requirements

[7] GOST 8.586.3-2005 (ISO 5167-3:2003) Measurement of Flowrate and Volume of Liquid and Gas by Means of Standard Pressure Differential Devices - Part 3. Nozzles and Venturi nozzles. Technical requirements

[8] GOST 8.586.4-2005 (ISO 5167-4:2003) Measurement of Flowrate and Volume of Liquid and Gas by Means of Standard Pressure Differential Devices - Part 4. Venturi tubes. Technical requirements

[9] GOST 8.586.5-2005 (ISO 5167-5:2003) Measurement of Flowrate and Volume of Liquid and Gas by Means of Standard Pressure Differential Devices - Part 5. Technique for making the measurements

[10] Pistun, Y. \& Lesovoi, L. Normalization of pressure differential flowmeters. // Publishing House of Institute of Energy Audit and Energy Carrier Accounting (www.ieoe.com.ua), ISBN 966-553541-2, Lviv - 2006, p. 576

[11] Pistun, Y.; Lesovoi, L.; Matiko, F.; Markovskyi, D \& Lesovoi, R. "RASKHOD-RU" CAD computer program. User manual. // Publishing House of Institute of Energy Audit and Energy Carrier Accounting (www.ieoe.com.ua), Lviv - 2007, p. 128 